\title{
COVID-19 impact on neurology training program in Italy
}

\author{
Francesco Di Lorenzo ${ }^{1}$ - Tommaso Ercoli ${ }^{2}$. Luca Cuffaro ${ }^{3}$. Francesco Barbato ${ }^{4}$. Francesco lodice ${ }^{5}$. \\ Gioacchino Tedeschi ${ }^{6}$ - Alessandro Bombaci ${ }^{7}$ - on behalf of SIgN
}

Received: 12 September 2020 / Accepted: 11 December 2020 / Published online: 14 January 2021

(C) Fondazione Società Italiana di Neurologia 2021

\begin{abstract}
The ongoing COVID-19 pandemic is having a huge impact on clinical activity of all hospitals, including the ones involved in training of residents. In addition, neurology residents underwent substantial modifications of their training program. Aim of our investigation was to evaluate the impact of COVID-19 pandemic on the educational activities of Italian neurology residents through an online questionnaire delivered to neurology residents. The results obtained showed that almost $30 \%$ of the respondents were redistributed to COVID-19 units. Neurology departments underwent substantial modifications of their organization influencing clinical educational activities; lessons and seminars were rescheduled online and research protocols were stopped and transferred to remote working, when feasible. There was a relevant use of telemedicine approach even if most of the respondents had never been trained before. Some of the changes had a North-South gradient, following the epidemiology of the pandemic. The data obtained from our survey highlight those points to address to be prepared for possible future emergencies.
\end{abstract}

Keywords COVID-19 $\cdot$ Italian resident $\cdot$ Neurology resident $\cdot$ Tele-health $\cdot$ Tele-neurology $\cdot$ SIgN $\cdot$ Neurology training · Psychological consequences

\section{Introduction}

The severe acute respiratory syndrome coronavirus 2 (SARS$\mathrm{CoV}-2$ ) is causing an ongoing pandemic of coronavirus disease 2019 (COVID-19) [1]. Italy has been one of the first countries to be involved in this pandemic, with a large hotbed

Alessandro Bombaci

ale.bombaci@gmail.com

1 Noninvasive Brain Stimulation Unit, Scientific Institute for Research, Hospitalisation and Health Care Santa Lucia Foundation, Rome, Italy

2 Department of Medical Sciences and Public health, Institute of Neurology, University of Cagliari, Cagliari, Italy

3 Department of Biomedicine, Neuroscience and Advanced Diagnostic, University Hospital "Paolo Giaccone", Palermo, Italy

4 IRCCS San Raffaele Cassino, Cassino, FR, Italy

5 Department of Neuroscience and Neurorehabilitation, IRCCS San Raffaele Pisana, Rome, Italy

6 Department of Medical and Surgical Sciences, University of Campania "Luigi Vanvitelli”, Naples, Italy

7 "Rita Levi Montalcini” Department of Neurology, University of Turin, Turin, Italy in Lombardia. On 7th March 2020, the central government called for a general lockdown with severe social restrictions across the whole country. Although neurologists were not strictly involved in the direct management of COVID-19positive patients at first, the devastating breadth of this situation caused profound changes also in the routine practice of neurological patient care and residency training. In light of the higher exposure to the virus of health care workers [2] and the operative strategies adopted for facing the emergency [3,4], it became mandatory a resettlement of the workforce with fast training sessions and new procedures to learn and protocols to respect. Moreover, due to the constantly increasing number of infected patients requiring clinical care and the social restrictions enforced (e.g., lockdown or social distancing), neurological activities in both outpatient and hospitalized patients underwent substantial changes. However, even if the pandemic did not spread uniformly (with a North to South negative gradient), the social limitations undertaken affected all the country to a certain extent.

As a young section of the Italian Society of Neurology [5] $(\operatorname{SIgN})$, we aimed at investigating the effects of COVID-19 pandemics on the educational activity of Italian neurology residents from both a clinical and a research point of view. 
Table 1 Demographic characteristics of neurology residents who completed the survey

\begin{tabular}{|c|c|c|c|c|}
\hline No. & $\operatorname{Sex}(M / F)$ & Age & $\begin{array}{l}\text { Distribution } \\
\text { (North/Center/ } \\
\text { South) }\end{array}$ & $\begin{array}{l}\text { No. per year of residence } \\
\text { (1st, 2nd, 3rd, 4th) }\end{array}$ \\
\hline 254 & $126 / 128$ & $29.3 \pm 2.3$ & $110 / 66 / 78$ & $36 / 68 / 94 / 56$ \\
\hline
\end{tabular}

\section{Design and setting}

We canvassed Italian neurology residents [6] using an online questionnaire.

In Italy, the Residency program in neurology is active in 36 teaching hospitals, and SIgN has a representative in each of the centers.

Before moving on we contacted the local SIgN representative asking seven questions (questions 1-7, see Table 1 in Supplementary Materials) concerning the general initiatives taken in their university hospital.

Then, a standardized email containing the link to the online questionnaire was sent to the SIgN representatives and to the directors of all the 36 schools on 30th April. We asked them to forward the email to trainees attending their neurology training program. The link to the online questionnaire was not publicly displayed through personal network or social media.

The survey was designed through Google survey by the five authors through online meetings and finalized by emails. No personal information was collected and no ethical approval was pursued. Second and third reminders to both representatives and directors were sent by email after 10 and 20 days. The survey was closed after 4 weeks. The questionnaire was composed by 22 items (see supplementary materials for the full questionnaire) in addition to questions about demographic data (gender, age, school of residency, year of training). We accepted as valid only the questionnaires in which all the questions were answered.

All the procedures were performed in accordance with the guidelines of good clinical practice for the realization of exploratory surveys [7].

\section{Results}

We received responses from 254 postgraduates (41\% of response rate) from 36 schools. Nineteen participants were spending a period abroad at the time of the COVID-19 outbreak. The mean age of participants was $29.3+2.3$ years and $50.2 \%$ of the residents were female. The demographic data of the participants are listed in Table 1.

\section{Lessons and seminars}

During this emergency, all lessons, seminars, and conferences were suspended in all Italian universities. In 33 out of 36 centers $(92 \%)$, lessons have been rescheduled on virtual platforms and in eight of them also seminars have been done online.

\section{Clinical activities}

Neurological wards underwent important changes in all Italian centers: most of the university neurological wards $(86 \%)$ interrupted the programmed admission, while the remaining (14\%) reduced its rate. The number of beds in neurology wards was reduced compared to the period prior the pandemic ( $81 \%$ of centers): up to $30 \%$ in $21 \%$ centers, up to $50 \%$ in $36 \%$ centers, and more than $50 \%$ in $24 \%$ centers.

Day hospital admissions, diagnostic exams, and intravenous infusion therapies were generally reduced too (Fig. 1b).

Programmed outpatients' visits were suspended during March and April in all Italian teaching hospitals; 1/3 of residents reported continuation of outpatient visits only for urgent management.

Almost all Italian residents who completed the survey (246; 97\%) underwent changes of their routine clinical activities (Fig. 1a), namely 138 (54\%) had a reduction of work shifts, of which $3 / 4$ (103) declared they only worked remotely. Of those who had to increase their work shifts $(63 ; 26 \%)$, half (31) also worked remotely. A tight connection between the increase of work shifts and the spread of COVID-19 has been observed through Italy (Fig. 3c). Almost half of the respondents $(113 ; 44 \%)$ have been divided in working groups to avoid increasing of contacts and exposure to the virus.

As far as the remote activities are concerned, $169(66 \%)$ of respondents reported that they were focused on preparation of clinical and scientific seminars and doing research (i.e., writing papers, dataset management, etc.) and 146 (57\%) of the participants helped in the remote management of both in- and outpatients.

The planned rotations in neurological and non-neurological wards have been largely modified (in $78 \%$ and $71 \%$ of the residents, respectively). Rotations in outpatient neurological services were interrupted in more than a half of cases because of temporary suspension of all the frontal clinics.

\section{Volunteers}

Seventy-two (28\%) of respondents were employed in COVID-19-dedicated units: most of them (81\%) were 
a Changes in clinical activities

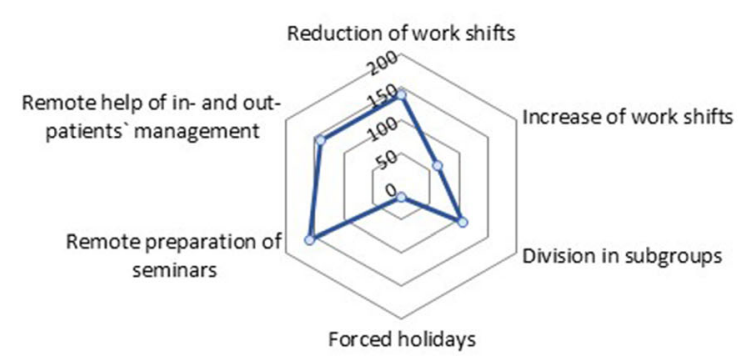

b Changes in day hospital activities

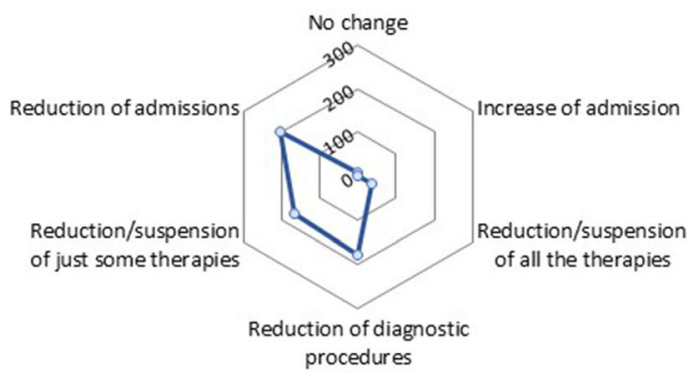

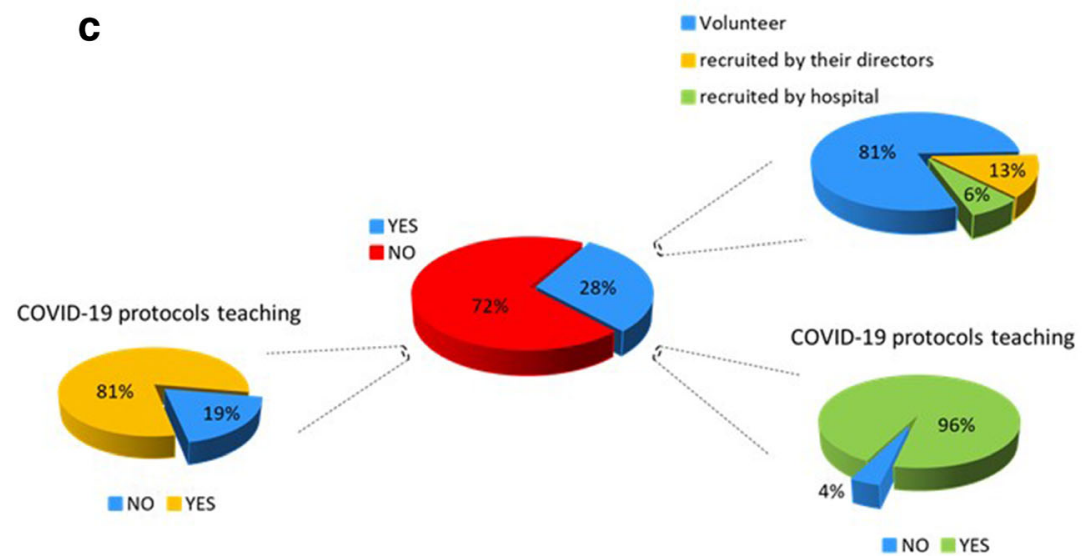

Fig. 1 Changes in hospital activities on neurology residents. a Changes in clinical activities include mainly reduction of work shift, division in subgroups, management of in- and outpatients remotely, and preparation of seminar. $b$ In day hospital, we observed a general

volunteers, $13 \%$ were recruited by their directors, and $6 \%$ were recruited directly by hospital (Fig. 1c). Before starting work in COVID facilities, $58 \%$ of them were trained in medical management of COVID-19 patients and $96 \%$ were extensively taught about the hygiene actions to undertake to contain the infection spreading. Focusing on volunteers, there was a clear decreasing gradient moving from North to Center to South (Fig. 3b).

\section{Research}

In more than half of the cases $(150 ; 58,6 \%)$, research activities were interrupted or reduced. Forty-one (16\%) respondents reported no changes in the amount of research activities, although most of the work was focused on database elaboration and on writing of reviews, while 36 respondents (14\%) reported an increase of research activities due to reduction of clinical activities was observed. Twenty-eight respondents (11\%) were not yet involved in research activities (most of them are attending first and second year of residency). Ninetyeight (43\%) of the residents who do research reported to have enough facilities to do research at home. Fifty-seven (25\% of cases) had difficulties to access scientific journals, while 25 (11\% of the residents) did not have adequate statistical

reduction of admission, therapies, and diagnostic procedures. c Around $28 \%$ of residents had to work in COVID-19 wards: most of them were volunteers $(81 \%)$. Teaching of COVID-19 protocols was fulfilled in almost all residents (96\%) who worked in COVID-19 wards

software at home to run analyses and 48 (21\% of the participant) did not have other tools fundamental for their research.

\section{Telemedicine}

The $35 \%$ of residents (90) declared that no instrument of telemedicine was adopted in their structures, while in $32 \%(82)$ it was employed only in case of urgency and in $29 \%$ (74) it was used for all outpatients (Fig. 2a). Ten of respondents (4\%) reported that in their structures telemedicine was employed also for first visits. The latter had a clear prevalence in North of Italy, followed, in order, by Center and by South of Italy.

Before the COVID-19 outbreak, tele-neurology was adopted only $14 \%$ by of the residents (36) and this was just once a month or less (Fig. 2b). Only $42 \%$ of residents (15), who already used tele-neurology before the pandemic, underwent a training course sponsored by their hospital.

\section{COVID-19 protocols}

Training and information by health care public bodies about protocols to adopt for containing COVID-19 spreading in hospitals (use of personal protective equipment, clothing, 
a

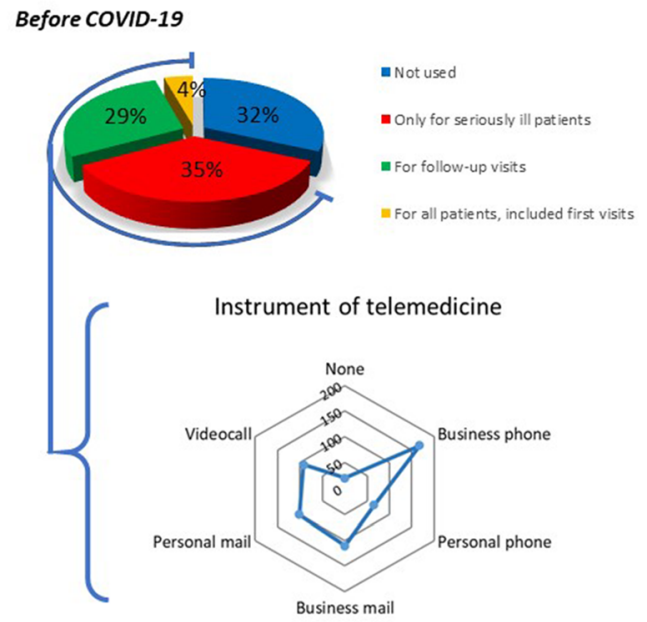

Fig. 2 Use of telemedicine during and before COVID-19 pandemic. During COVID-19 pandemic, we observed a wide use of telemedicine: most of ambulatorial services used it to visit seriously ill patients and, some of them, also to do follow-up visits (a). Most important instruments

masks, hand-wash, etc.) was obtained in 216 (85\%) of respondents (Fig. 1c).

These data showed no regional differences through Italy. Most of training $(166 ; 77 \%)$ was based on online teaching material. One hundred fifteen of respondents $(45 \%)$ fulfilled a medical training focused on management of COVID-19 patients.

\section{Psychological implications}

Two hundred forty of respondents (94\%) reported a worsening of psycho-physical conditions of neurological patients, and only $36(14 \%)$ reported to be self-confident enough to guarantee an appropriate psychological support.

Finally, a psychological support for residents working during the pandemic outbreak was offered to 64 neurology residents (26\%) of all cases and it showed a clear North-CenterSouth decreasing gradient (Fig. 3d).

\section{Discussion}

The ongoing COVID-19 pandemic induced many changes of the routine activities among neurology residents. Our survey aimed at investigating the main consequences of the pandemic on neurology training program to identify the criticalities resulting in the management of such outbreak. Even though the situation differed within our country and induced the developing of different solutions proportional to the gravity of the pandemic spread within a certain area, the substantial changes caused by the pandemic in social life (i.e., lockdown, social distancing, and prohibition of visitors in hospitals) were similar all along the national territory. Indeed as it can be b

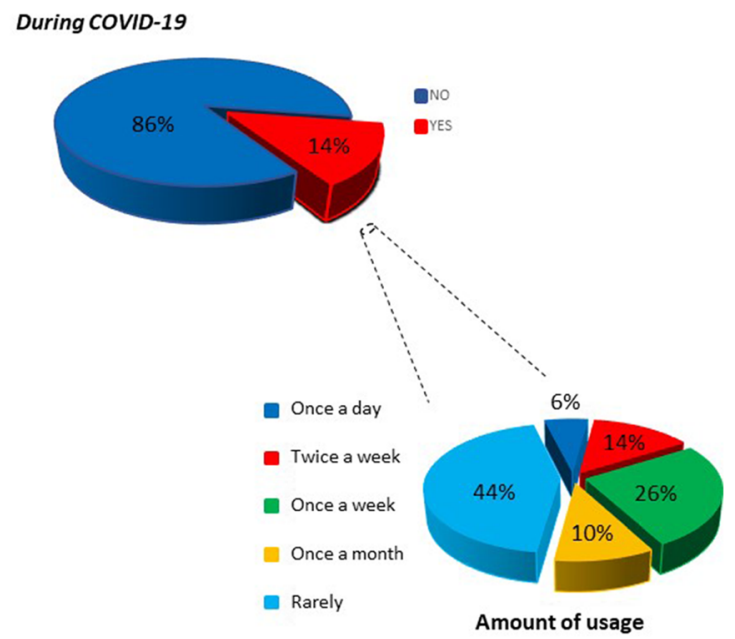

of telemedicine were business phone and mail. We can highlight a clear change from the past when telemedicine was used only by $14 \%$ of neurology resident (b). Moreover, the latter used telemedicine rarely or only few times per week

observed in Fig. 3, much of the differences observed in participants' replies, in terms of geographical distribution, rely on the specific involvement of the training centers during the pandemic at the time of our survey. The gradient NorthSouth observed in our data includes just practical aspects induced by the gravity of the pandemic.

The sudden need for beds in COVID-19 facilities demanded to meet the huge number of infected patients and the growing exposure of health care workers to the virus has altered the normal organization of hospitals. Consequently, to provide sufficient capacity for potential COVID-19 patients, we observed a dramatic reduction of hospital beds in neurological wards in concomitance with a quite complete cancellation of elective hospitalization of patients suffering from neurological disorders. These circumstances, along with reduction of admissions to day hospital and suspension of some therapies or diagnostic procedures, deprived residents from an important source of clinical educational activity such as bedside teaching [8].

The attendance in neurological specialized clinic included neurophysiological services, so that even this remarkable experience has been interrupted, due to suspension of routine activities.

The social restrictions adversely affected the planning and the management of outpatient care [9]. Most hospitals postponed outpatient appointments for new referrals and patients were examined just in case of an emergency referral. Urgent consultations of patients requiring specific care (e.g., patients with Parkinson's disease, multiple sclerosis, or epilepsy) were managed via telemedicine. It is well known that all chronic neurological conditions require regular and punctual followup; therefore, it has been challenging to take care of outpatients, especially via telemedicine. Importantly, telemedicine visits might be complicated for doctors and patients not used 


\section{a Total cases of COVID-19 in Italy}

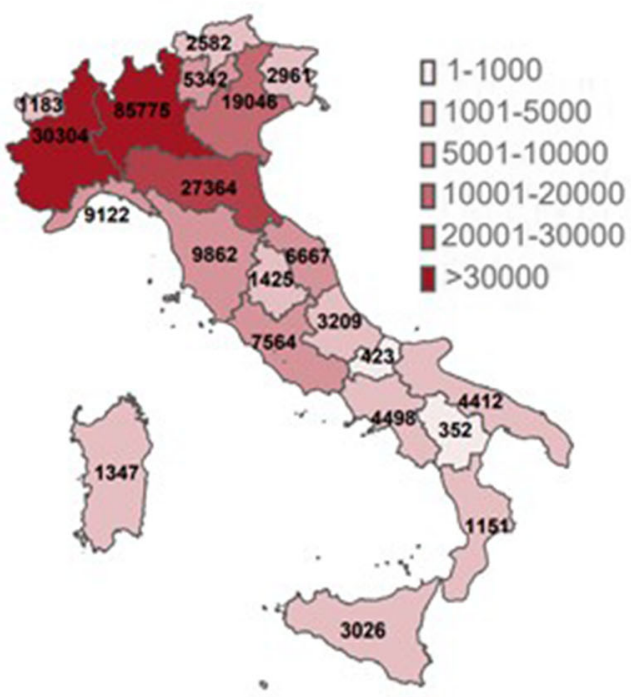

C
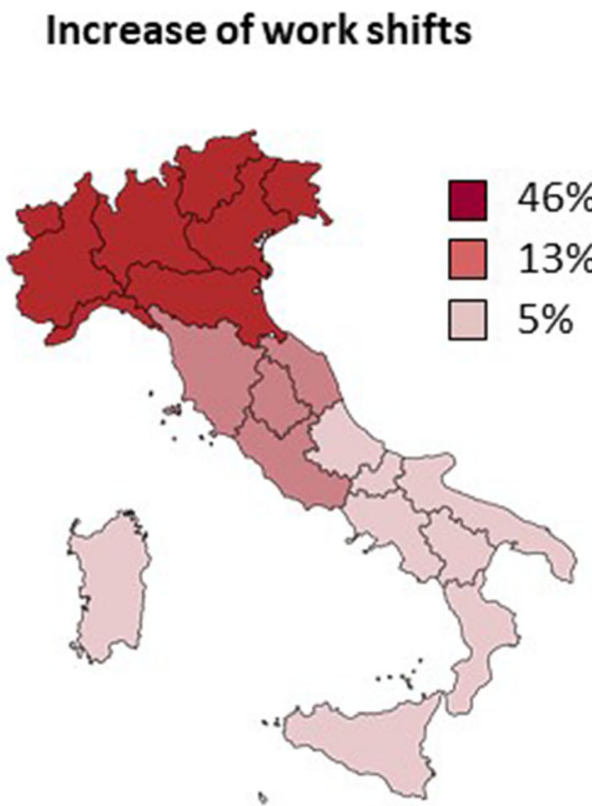

Fig. 3 Correlation between COVID-19 pandemic spreading through Italy and changes during COVID-19 pandemic. a Distribution of total ascertained cases of COVID-19 in Italy up to May 21st. b We observed a clear North-Center-South decreasing gradient of residents who decided to go volunteer in neurological districts. c Also the increase of work shift

to them, hampering the quality of the visit outcome: indeed, we have to take into account that most of neurological patients are aged and could not be familiar with modern technologies and some of them have cognitive impairment $[10,11]$.

The COVID-19 pandemic has catalyzed the use of digital technologies for the care of neurologic patients $[12,13]$. The data from our survey showed that in few centers the use of telemedicine consultations or digital technologies was a

\section{b Volunteers in COVID-19 districts}

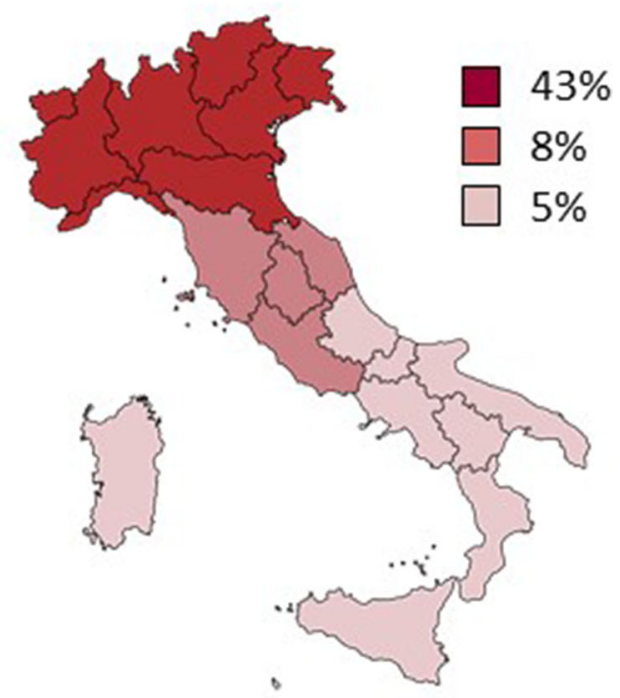

d Psychological support

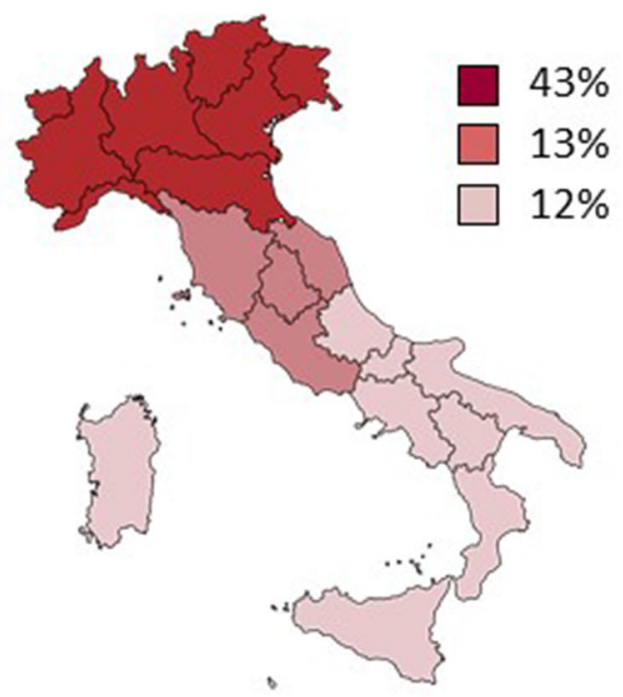

was observed mainly in almost half of residents who were working in the North during COVID-19 pandemic. d A psychological support was fulfilled in $43 \%$ of residents who lived in the North, in $13 \%$ in the Center, and in $12 \%$ in the South, reproducing the gradient of COVID19 spreading

common practice before the COVID-19 pandemic, without geographical and sub-specialization differences, and also that just few residents had been adequately trained for this purpose.

Importantly, we found that in almost all the neurology teaching hospitals the theoretical educational activities were not halted by the pandemic. After a first period of interruption, indeed within 5 weeks lessons started again remotely and, in some cases, also interdisciplinary seminars were given. 
The current situation is compromising also research and clinical trials. Most of the projects have been somehow interrupted and all the studies conducted during this period will need to be carefully interpreted. Even if it is possible to work remotely on research projects, especially for data analysis, or manuscript drafting, the lack of proper tools has limited the productivity of residents in accomplishing their research objectives.

In the most affected areas, the increasing number of COVID-19 patients and the lack of medical staff due to infected colleagues or quarantine induced deep changes also to the management of the workforce. As most of the bed capacity was converted to the care of this emergency, many doctorsof varying disciplines - have been forced to work outside of their field (e.g., in internal medicine, intermediate or intensive care units) and very often they had to face a dramatic increase of working hours. The daily routine has been entirely upended by new measures to internalize, longer shifts, irregular and flexible schedules, and expected continuous availability.

However, it has to be noted that the reduction of the abovementioned clinical activities caused a paradoxical diminished request for medical staff not involved in COVID19 activities. Consequently, some residents were put on standby. While immediate workload reduction to those sent home might seem appealing, the continuous availability and uncertainty has a significant impact on personal freedom in addition the generally implemented social restrictions.

Another important issue to take into account is the fact that patients were not allowed to have visitors (or in some cases just one visitor a day), so neurologists and residents were more frequently faced with dispirited patients and need to provide more psychological care. For chronic neurological patients, the social restrictions had an important impact on their lives, and psychological consequences may have worsened their quality of life [14]. The lesson we have learned is that more space should be given during the training program to the acquisition of communication and psychological skills to reduce the burden of afflicted patients [15].

Quick training sessions, new procedures and protocols, and the constant change of information, protocols, and schedules put particularly unexperienced residents under significant pressure. However, our data show that in the most affected areas adequate psychological support was given to residents in need.

In conclusion, the data of our survey outlined that during this period neurology residents had to face several changes. In the present study, we identified several critical issues and we would conclude that neurology training should be improved in such points: (i) training telemedicine and the new digital technologies; (ii) strengthen psychological education, for a better management of patients in need; and (iii) improvement of the research tools (i.e., remotely papers access, statistical software, etc.).
It is important to take note of all the difficulties encountered during last months in all the aspects of neurology education in order to better tailor residents' preparation, especially in light of possible future seasonal outbreaks of COVID-19 [16] or large-scale emergencies.

Supplementary Information The online version contains supplementary material available at https://doi.org/10.1007/s10072-020-04991-5.

Data availability Not applicable.

\section{Compliance with ethical standards}

Conflict of interest The authors declare that they have no conflict of interest.

Ethical approval None.

Code availability Not applicable.Supplementary Information The online version contains supplementary material available at https://doi. org/10.1007/s10072-020-04991-5.

\section{References}

1. World Health Organization (2020). Surveillance case definitions for human infection with novel coronavirus $(\mathrm{nCoV})$ : interim guidance v1, January 2020. World Health Organization. https://apps. who.int/iris/handle/10665/330376. License: CC BY-NC-SA 3.0 IGO

2. Zhang $\mathrm{H}$ (2020) Early lessons from the frontline of the 2019-nCoV outbreak. Lancet 395:687

3. Grasselli G, Pesenti A, Cecconi M (2020) Critical care utilization for the COVID-19 outbreak in Lombardy, Italy: early experience and forecast during an emergency response. JAMA. 323:15451546. https://doi.org/10.1001/jama.2020.4031

4. Spina S, Marrazzo F, Migliari M, Stucchi R, Sforza A, Fumagalli R (2020) The response of Milan's Emergency Medical System to the COVID-19 outbreak in Italy. Lancet 395(10227):e49-e50

5. Di Lorenzo F, Alberti P, Pavolucci L, Pietrafusa N, Iodice F (2019) Introduction to the Italian section for young neurologists. Neurol Sci. 40(11):2423-2424

6. Iodice F, Brunetti V, Nicolini E, Paolucci M, Di Lorenzo F (2019) Endovascular therapy for acute ischemic stroke: which role for neurologists? Neurol Sci. 40(8):1737-1739

7. Kelley K, Clark B, Brown V, Sitzia J (2003 Jun) Good practice in the conduct and reporting of survey research. Int J Qual Health Care. 15(3):261-266

8. van der Meulen M, Kleineberg NN, Schreier DR, García-Azorin D, Di Lorenzo F (2020 Dec) COVID-19 and neurological training in Europe: from early challenges to future perspectives. Neurol Sci. 41(12):3377-3379

9. Caso V, Federico A (2020) No lockdown for neurological diseases during COVID-19 pandemic infection. Neurol Sci. 41(5):9991001. https://doi.org/10.1007/s10072-020-04389-3

10. Cuffaro L, Di Lorenzo F, Bonavita S, Tedeschi G, Leocani L, Lavorgna L (2020) Dementia care and COVID-19 pandemic: a necessary digital revolution. Neurol Sci. 41(8):1977-1979. https:// doi.org/10.1007/s10072-020-04512-4 
11. Miele G, Straccia G, Moccia M et al (2020) Telemedicine in Parkinson's disease: how to ensure patient needs and continuity of care at the time of COVID-19 pandemic [published online ahead of print, $2020 \mathrm{Jul}$ 13]. Telemed J E Health. https://doi.org/10.1089/ tmj.2020.0184

12. Bloem BR, Dorsey ER, Okun MS (2020) The coronavirus disease 2019 crisis as catalyst for telemedicine for chronic neurological disorders [published online ahead of print, 2020 Apr 24]. JAMA Neurol. https://doi.org/10.1001/jamaneurol.2020.1452

13. Hatcher-Martin JM, Adams JL, Anderson ER, Bove R, Burrus TM, Chehrenama M, Dolan O’Brien M, Eliashiv DS, Erten-Lyons D, Giesser BS, Moo LR, Narayanaswami P, Rossi MA, Soni M, Tariq N, Tsao JW, Vargas BB, Vota SA, Wessels SR, Planalp H, Govindarajan R (2020) Telemedicine in neurology: Telemedicine Work Group of the American Academy of Neurology update. Neurology 94(1):30-38. https://doi.org/10.1212/WNL. 0000000000008708
14. Bhaskar S, Bradley S, Israeli-Korn S et al (2020) Chronic neurology in COVID-19 era: clinical considerations and recommendations from the REPROGRAM consortium. Front Neurol 11:664. Published 2020 Jun 24. https://doi.org/10.3389/fneur.2020.00664

15. Willis R, Strowd RE, Barks MC, Salas RE, Gamaldo CE, Lemmon ME (2020) Education research: the medical student perspective on challenging conversations. Neurology. 95(5):226-230

16. Kissler SM, Tedijanto C, Goldstein E, Grad YH, Lipsitch M (2020) Projecting the transmission dynamics of SARS-CoV-2 through the postpandemic period. Science 368(6493):860-868. https://doi.org/ 10.1126/science.abb5793

Publisher's note Springer Nature remains neutral with regard to jurisdictional claims in published maps and institutional affiliations. 